Disclosure of Interest: None declared

DOI: 10.1136/annrheumdis-2017-eular.6970

\section{FRI0271 PREVALENCE AND ASSOCIATED FACTORS OF DEPRESSIVE DISORDERS IN CHINESE PATIENTS WITH SYSTEMIC LUPUS ERYTHEMATOSUS (SLE)}

C.C. Mok ${ }^{1}$, L.Y.T. Lo ${ }^{2}$, K.S. Poon ${ }^{2}$, C.W. Cheng ${ }^{2} .{ }^{1}$ Medicine, Tuen Mun Hospital; ${ }^{2}$ Psychiatry, Castle Peak Hospital, HK, Hong Kong

Background: Psychiatric symptoms are common in patients with SLE. Most studies utilized self-rated scales of psychiatric symptoms for evaluation. Formal diagnosis of depression was not established by psychiatric interviews.

Objectives: To determine the prevalence of depressive disorders, severity of depressive symptoms and the associated clinical and socioeconomic factors in Chinese patients with SLE.

Methods: Patients who fulfilled $\geq 4$ ACR criteria for SLE were randomly recruited from rheumatology out-patient clinics and hospital admission in a 9-month period. Psychiatric disorders were diagnosed by a direct interview with the psychiatrist using the Chinese-bilingual Structural Clinical Interview for DSM-IV Axis I disorders, patient research version (CB-SCID-I/P). The severity of depressive symptoms was assessed by the validated Chinese Hamilton Depressive Rating Scale (HAM-D). Patients were also asked to complete the Beck Depression Inventory (BDI), Medical Outcomes Study Social Support Survey (MOS-SSS-C) and the WHO Quality of Life Measure-Abbreviated Version (WHOQOL-BREF, $\operatorname{BREF}(\mathrm{HK})$ ). SLE disease activity (SLEDAI), organ damage (SLICC/SDI) and socio-demographic were collected and correlated with the presence of psychiatric disorders. Logistic regression models were used to study the independent factors associated with depressive disorders and the severity of depressive symptoms.

Results: 175 SLE patients were studied (95\%women, age $39.2 \pm 12.4$ years, SLE duration $10.3 \pm 6.7$ years). $27(15 \%)$ and $37(21 \%)$ patients were diagnosed with a current depressive (52\%major depressive disorder, $22 \%$ dysthymia) or anxiety (35\%generalized anxiety, 14\%panic, 14\%phobia, 8\%adjustment) disorders, respectively. Patients with depressive disorders, as compared to those without psychiatric disorders, had more active SLE $(p=0.03)$ and were more likely to have a history of psychiatric diagnosis $(p<0.001)$ and financial assistance from Government $(\mathrm{p}=0.04)$. Independent factors associated with a depressive disorder were SLEDAI score (1.13 [1.02-1.24]; $p=0.02)$, perceived poor social support $(p=0.03)$ and a past history of psychiatric disorders $(p=0.003)$. Age, disease duration and other socio-economic variables such as educational level and marriage status were not correlated with the presence of a depressive disorder. Being separated/divorced $(\beta=0.19 ; p=0.02)$, having a higher SLEDAI score $(\beta=0.16 ; p=0.02)$, SLE duration $(\beta=-0.18 ; p=0.02)$ and a past history of psychiatric disorders $(\beta=0.18 ; p=0.01)$ were independently associated with higher HAM-D scores, which reflect more severe depressive symptoms. Depressive disorders and severity of depressive symptoms were significantly associated with poorer quality of life. ROC analysis showed that a cut-off of 14 points of the self-rated BDI had a sensitivity of $89 \%$ and a specificity of $83 \%$ for differentiating a current depressive disorder from those without.

Conclusions: A diagnosis of depressive disorders is prevalent in Chinese patients with SLE. Independent risk factors are more active disease, perceived poor social support and a past history of psychiatric disorders. Patients with more active SLE, shorter disease duration, a past history of psychiatric disorders and being separated were associated with more serious depressive symptoms. The self-rated BDI provides a good screening tool for identifying depressive disorders in SLE patients.

Disclosure of Interest: None declared

DOI: 10.1136/annrheumdis-2017-eular.3935

\section{FRI0272 EARLY DAMAGE ASSESSMENT AND PREDICTION OF DAMAGE ACCRUAL IN PRIMARY SJÖGREN'S SYNDROME USING SALIVARY GLAND ULTRASONOGRAPHY DURING 2 YEARS OF FOLLOW-UP}

C. Baldini $^{1}$, N. Luciano ${ }^{1}$, C. Seghieri ${ }^{2}$, F. Ferro ${ }^{1}$, E. Elefante ${ }^{1}$, M. Mosca $^{1}$. ${ }^{1}$ Clinical and Experimental Medicine, Rheumatology Unit, University of Pisa ${ }^{2}$ Laboratorio Management e Sanità, Scuola Superiore Sant'Anna, Pisa, Italy

Background: Early detection and prediction of glandular damage in primary Sjögren's syndrome (pSS) is of pivotal importance in patients' stratification and treatment. Recently, ultrasonography (SGUS) has appeared as a promising tool for the assessment of salivary gland involvement in pSS. However, few studies have specifically explored its role in the early identification of salivary gland damage and in monitoring disease progression over the follow-up.

Objectives: a) to explore the contribution of SGUS in the early assessment of pSS-induced glandular damage and in monitoring damage accrual during the follow-up b) to identify predictive factors associated with the development and progression of sonographic changes in salivary glands.

Methods: An inception cohort of 54 pSS (AECG 2002) patients was included in this study at the diagnosis and prospectively followed after 6,12 and 24 months. Demographic, clinical data and the ESSDAI of the patients were collected at each study visit as well as SGUS score that was calculated every time by the same radiologist. Sonographic assessment of glandular damage was performed by evaluating the size of the parotid and submandibular glands (normal/reduced) and the presence/absence of hyperechoic bands in more than $50 \%$ of the glands (i.e fibrosis). Descriptive statistics and logistic regression were used for the data analysis.

Results: We included 54 patients $(51 \mathrm{~F}: 3 \mathrm{M})$ with a median duration of symptoms before diagnosis of 11 months (range $36-5 \mathrm{mo}$ ) and a median (IQR) ESSDAI at baseline of $5(1-8)$. We found that at baseline 13 patients out of 54 $(24.1 \%)$ already presented one element of damage in their SGUS evaluation, $14 / 54(25.9 \%)$ more than one element of damage, whereas only $27 / 54(50 \%)$ of patients did not present any sonographic sign suggestive for damage. In particular, hyperechoic bands were detected in the parotid glands of $12 / 54(22 \%)$ patients and in the submandibular glands of $21 / 54(38.9 \%)$; reduced parotid and submandibular gland size were described in 5/54 (9.3\%) and in 10/54 (18.5\%) of the cases, respectively. Predictive factors associated with sonographic salivary gland damage at the diagnosis were the ESSDAI at baseline $(\mathrm{OR}=1.13[95 \% \mathrm{Cl}$ 1.0 to 1.27$], p=0.04)$ and the positivity for Rheumatoid factor (RF) (OR $=4.47$ [95\% $\mathrm{Cl} 1.29$ to 15$], \mathrm{p}=0.02)$. During the follow-up, 15/54 (27.8\%) patients presented a progression of their salivary gland damage. We specifically observed a significant increase in the frequency of reduced parotid $(9.3 \%$ vs $25.9 \%, p=0.004)$ and submandibular gland size $(18.5 \%$ vs $33.3 \%, p=0.008)$ at the end of follow-up. Predictive factors for damage accrual during follow-up were: minor salivary gland focus score ( $\mathrm{OR}=3.5$ [95\% $\mathrm{Cl} 1.1$ to 10.6$], \mathrm{p}=0.03$ ) and the ESSDAI at baseline (OR $=1.64$, [95\% $\mathrm{Cl} 1.0$ to 2.6$] \mathrm{p}=0.03$ ).

Conclusions: Sonographic assessment of glandular damage apparently revealed a relative high frequency of already established signs of glandular damage in pSS patients at baseline, especially in patients with positive RF and higher ESSDAI. When routinely used, SGUS may accurately allowed to monitor damage accrual over the follow-up, ultimately contributing to a better clinical management of patients.

Disclosure of Interest: None declared

DOI: 10.1136/annrheumdis-2017-eular.4327

\section{FRI0273 ROLE OF PROCALCITONIN AND C-REACTIVE PROTEIN IN SCREENING OF INFECTION IN PATIENTS WITH SYSTEMIC LUPUS ERYTHEMATOSUS}

C.H. Ho, A.M.H. Leung. Department of Medicine, Queen Elizabeth Hospital, Kowloon, Hong Kong

Background: Previous studies revealed conflicting results regarding the role of procalcitonin in predicting infections in patients with systemic lupus erythematosus (SLE).

Objectives: This retrospective study aimed to analyse the role of procalcitonin (PCT) and C-reactive protein (CRP) in predicting infections in SLE patients, and to determine an optimal cut-off value for PCT and CRP for defining sepsis.

Methods: This study was carried out in one single tertiary centre. Adult patients (>18-year-old) with underlying SLE who were admitted to hospital between 1st Jan 2007 and 31st Dec 2015 were included. Demographic data, PCT and CRP upon admission, and other clinical parameters were obtained. Infection was defined by positive culture, or based on clinical and radiological evidence with subsequent response to antimicrobial treatments. Active SLE disease activity was defined by SLEDAI, and also by SLE-related manifestations not included in SLEDAI. Mann-Whitney test was used to test the difference between numerical parameters between patients with and without infection. Spearman's correlation was used to analyse the correlation between PCT and CRP. Receiver operating characteristic $(R O C)$ curves were plotted to define an optimal cut-off values for PCT and CRP in infection.

Results: 33 (27 female \& 6 male) SLE patients were included. Both mean and median age were 42-year-old. Among the 21 septic patients, 9 had active lupus and 12 had inactive disease. All but one of the 12 non-septic patients had active lupus. All 4 patients with underlying renal failure belonged to the infection group. There were 13 and 3 patients on immunosuppressive treatments in infection and non-infection groups respectively.

In patients with infection, mean PCT was $5.74 \mathrm{ng} / \mathrm{ml}$ and mean CRP was $77.22 \mathrm{mg} / \mathrm{L}$. In those without, these were $0.29 \mathrm{ng} / \mathrm{ml}$ and $20.04 \mathrm{mg} / \mathrm{L}$ respectively. Both PCT $(p=0.014)$ and CRP $(p=0.016)$ levels were significantly higher in patients with infection than those without. There was no significant difference between the PCT and CRP levels in both septic patients (PCT $p=0.862$, CRP $p=0.247$ ) and non-septic patients (PCT $p=1.000, C R P p=0.500$ ) regardless of their SLE disease activity.

PCT level correlated positively with CRP level $(r=0.456, p=0.008)$, but it had no correlation with age $(p=0.978)$, gender $(p=0.424)$, underlying renal failure $(p=0.304)$, steroid $(p=0.053)$ or other immunosuppressants $(p=0.132)$ use.

ROC curves of PCT and CRP showed a similar area under curve (AUC) of 0.756 and 0.752 respectively. The cut-off for PCT was $0.78 \mathrm{ng} / \mathrm{ml}$ (sensitivity $61.9 \%$, specificity $91.7 \%$ ), giving a positive predictive value (PPV) of $92.9 \%$. The cut-off for CRP was $9.35 \mathrm{mg} / \mathrm{l}$ (sensitivity $85.7 \%$, specificity $66.7 \%$ ), giving a PPV of $81.8 \%$. Combining both PCT and CRP above their cut-offs, the specificity of predicting infection improved to $100 \%$ but the sensitivity worsened to $52 \%$.

Conclusions: Both PCT and CRP were useful in predicting infection SLE patients regardless of their disease activity. The cut-off of PCT at $0.78 \mathrm{ng} / \mathrm{ml}$ and CRP at $9.35 \mathrm{mg} / \mathrm{l}$ gave satisfactory positive predictive value for infection. 